\title{
Medida Interpessoal de Psicopatia (IM-P): estudo preliminar no contexto brasileiro
}

\author{
Interpersonal Measure of Psychopathy (IM-P): \\ preliminary study in the Brazilian context
}

Tárcia Rita Davoglio, Gabriel José Chittó Gauer², Silvio José Lemos Vasconcellos ${ }^{3}$, Guinter Lühring ${ }^{4}$

\begin{abstract}
Resumo
Introdução: A observação direta do comportamento interpessoal é um recurso importante na descrição e diagnóstico da personalidade psicopática. A Medida Interpessoal de Psicopatia (Interpersonal Measure of Psychopathy, IM-P) é um instrumento psicométrico composto por 21 itens, desenvolvido para ser utilizado em associação com outras escalas de avaliação da psicopatia. Foca-se, especificamente, nos comportamentos interpessoais e aspectos não verbais evidentes na interação do entrevistador com indivíduos que apresentam características psicopáticas.

Objetivo: Descrever resultados preliminares sobre a investigação de aspectos interpessoais da psicopatia mediante a utilização da IM-P, incluindo as etapas de tradução/adaptação e avaliação de confiabilidade interavaliadores da IM-P, em uma amostra de adolescentes brasileiros.

Método: Trata-se de estudo transversal, descritivo e correlacional realizado com 20 adolescentes masculinos cumprindo medida socioeducativa com privação de liberdade na Região Metropolitana de Porto Alegre (RS). Após os procedimentos de tradução da escala, treinamento dos pesquis adores e teste piloto por meio de uma entrevista semiestruturada, a IM-P foi pontuada por três juízes independentes. Resultados: Os resultados estatísticos, obtidos através do coeficiente de concordância de Kendall, revelaram grau de concordância interavaliadores elevado e satisfatório para os escores totais da IM-P $(\mathrm{W}=0,84 ; \mathrm{p}<0,001)$. Alguns itens da escala que apresentaram resultados discrepantes foram discutidos e avaliados isoladamente. Conclusão: Do ponto de vista das propriedades psicométricas da versão em português da IM-P, os resultados deste estudo, embora incipientes, indicaram que a concordância interavaliadores foi consistente e que a escala se mostrou confiável. As limitações do estudo são apresentadas, bem como alguns questionamentos relevantes à continuidade da pesquisa com a IM-P. Descritores: Transtornos da personalidade, relações interpessoais, avaliação, psicometria.
\end{abstract}

\begin{abstract}
Introduction: The direct observation of interpersonal behaviors is an important resource in the description and diagnosis of the psychopathic personality. The Interpersonal Measure of Psychopathy (IM-P) is a psychometric instrument comprised of 21 items, designed to be applied in association with other instruments that also evaluate psychopaths. It focuses specifically on interpersonal and non-verbal behaviors that become evident during the interaction between the interviewer and individuals with psychopathic characteristics.

Objective: To describe preliminary results obtained in the assessment of interpersonal behaviors of psychopathic individuals using the IM-P, including instrument translation/adaptation and inter-rater reliability results, in a sample of Brazilian adolescents. Method: This was a cross-sectional, descriptive, correlation study conducted with a sample of 20 institutionalized male adolescents from the metropolitan area of Porto Alegre, state of Rio Grande do Sul, southern Brazil. Following instrument translation, interviewer training, and a pilot study conducted using a semi-structured interview, the IM-P was scored by three independent raters.

Results: The statistical results obtained using Kendall's coefficient of concordance revealed a high and satisfactory inter-rater agreement for IM-P total scores $(\mathrm{W}=0.84 ; \mathrm{p}<0.001)$. Some items showed conflicting results and were therefore discussed and evaluated separately.

Conclusion: The psychometric properties of the Brazilian Portuguese version of the IM-P, as assessed in this preliminary study, showed consistent inter-rater concordance, suggesting instrument reliability. Some study limitations are discussed, as are some relevant aspects that should be taken into consideration in future research involving the IM-P.
\end{abstract}

Keywords: Personality disorders, interpersonal relations, assessment, psychometrics.

${ }_{1}^{1}$ Psicóloga. Doutoranda em Psicologia Clínica, Programa de Pós-Graduação em Psicologia (PPGP), Pontifícia Universidade Católica do Rio Grande do Sul (PUCRS), Porto Alegre, RS. ${ }^{2}$ Psiquiatra. Professor/pesquisador, PPGP e Programa de Pós-Graduação em Ciências Criminais, PUCRS. ${ }^{3}$ Psicólogo. Professor, Departamento de Psicologia, Universidade Federal de Santa Maria (UFSM), Santa Maria, RS. ${ }^{4}$ Psicólogo. Mestre em Psicologia Clínica, PUCRS.

Apoio financeiro: Conselho Nacional de Desenvolvimento Científico e Tecnológico (CNPq; bolsa produtividade n 301885/2007 e 00659/2010-5) e Coordenação de Aperfeiçoamento de Pessoal de Nível Superior (CAPES).

Recebido em 07/02/2011. Aceito em 30/05/2011. Não foram declarados conflitos de interesse associados à publicação deste artigo.

Como citar: Davoglio TR, Gauer GJC, Vasconcellos SJL, Lühring G. Medida Interpessoal de Psicopatia (IM-P): estudo preliminar no contexto brasileiro. Trends Psychiatry Psychother. 2011;33(3):147-55. 


\section{Introdução}

Na atualidade, a psicopatia é considerada uma forma específica e extrema de transtorno da personalida$\mathrm{de}^{1}$. A essência do transtorno se manifesta principalmente nas relações interpessoais disfuncionais ${ }^{2}$, sendo identificada não apenas pelos aspectos transgressores e criminosos, mas, sobretudo, por uma série de características afetivas, interpessoais e comportamentais definidas por $\mathrm{Cleckley}^{3}$ e revistas por Hare ${ }^{4,5}$.

O indivíduo com esse transtorno apresenta, então, um estilo interpessoal enganador e arrogante, desinibido, egocêntrico e autoengrandecido, levando-o a mentir, trapacear e manipular facilmente ${ }^{5}$. Suas experiências afetivas são consideradas deficitárias, com pouca capacidade para sentir remorso, culpa e empatia, tendendo para a insensibilidade e incapacidade de aceitar responsabilidade pelas ações praticadas. Já o comportamento se mostra impulsivo ou irresponsável, com busca contínua por estimulação, falta de metas a longo prazo, falha em pensar antes de agir e um estilo de vida parasita 6 .

A psicopatia vem sendo frequentemente avaliada em contextos clínicos e forenses ${ }^{7}$, inclusive com populações adolescentes ${ }^{8}$, com significativo aumento da atenção para as metodologias de avaliação do construto ${ }^{9}$. Contudo, a avaliação dos aspectos interpessoais da psicopatia mostra-se mais complexa do que a avaliação dos aspectos comportamentais, sociais ou criminais ${ }^{2}$.

O comportamento interpessoal é um aspecto crucial na descrição e diagnóstico de todos os transtornos da personalidade ${ }^{10}$, incluindo a psicopatia ${ }^{2}$. Porém, a crítica principal dirigida à maioria dos estudos que investiga a associação entre os transtornos de personalidade e o comportamento interpessoal é a utilização exclusiva de medidas retrospectivas de autorrelato ${ }^{11}$. Uma revisão da literatura proposta por Klonsky et al. ${ }^{12}$ concluiu que os indivíduos com transtornos de personalidade são frequentemente inábeis para se ver realisticamente e para avaliar adequadamente o impacto de seu comportamento sobre as outras pessoas. Tendem, assim, a engrandecer ou minimizar suas responsabilidades, o que torna seu autorrelato pouco fidedigno para pesquisa.

De acordo com Oltmanns et al. ${ }^{13}$, em situações de avaliação, visando contornar tais dificuldades, tornou-se comum obter informações colaterais através de parentes ou amigos que conhecem bem o sujeito pesquisado. No entanto, segundo os referidos autores, haveria forte probabilidade de os padrões para julgamento e comportamento interpessoal do informante não serem confiáveis e fidedignos, podendo o relacionamento entre esses indivíduos já estar afetado pela presença do transtorno.
Em decorrência, a observação direta do comportamento interpessoal ${ }^{11}$ é apontada como um recurso importante na pesquisa dos transtornos que envolvem a personalidade. A observação e identificação de comportamentos específicos em situações relevantes para o funcionamento interpessoal adaptável pode ser útil para compreender as dificuldades interpessoais que se repetem e que sustentam esses transtornos ${ }^{14}$. Acredita-se, assim, que é possível fazer inferências acerca da personalidade baseando-se no comportamento interpessoal de uma pessoa durante a observação da sua interação com os outros².

Partindo desses pressupostos, foi desenvolvida a Medida Interpessoal de Psicopatia (Interpersonal Measure of Psychopathy, IM-P) ${ }^{2}$, que visa avaliar especificamente os comportamentos interpessoais e aspectos não verbais das interações sugeridos como típicos nas entrevistas com indivíduos que apresentam características psicopáticas. A IM-P se foca diretamente nas interações interpessoais reais que se estabelecem entre 0 examinando e o entrevistador. Segundo seus autores, a medida parte do princípio de que a avaliação direta de comportamentos evidentes em uma situação específica, a entrevista, reduz a necessidade de julgamentos subjetivos na medição de aspectos interpessoais da psicopatia. Ou seja, diante do fato de a entrevista contar com a participação do sujeito supostamente psicopata, seria possível detectar evidências das características disfuncionais por meio de suas atitudes e reações direcionadas ao entrevistador.

A IM-P vem sendo utilizada em estudos interna-

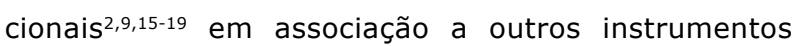
que avaliam a personalidade psicopata, especialmente o Inventário de Psicopatia de Hare Revisado (Hare Psychopathy Checklist Revised, $\mathrm{PCL}-\mathrm{R})^{5}$, nas suas diversas versões. Com populações adolescentes, tem sido utilizado o Inventário de Psicopatia de Hare Versão Jovens (Hare Psychopathy Checklist - Youth Version, PCL:YV) ${ }^{8}$, instrumento desenvolvido a partir de avanços e adaptações relacionadas à avaliação da psicopatia em adultos. Na última década, pesquisas similares vêm sendo introduzidas no Brasil, considerando tanto as peculiaridades dos estudos empíricos com novos instrumentos de avaliação ${ }^{20}$ quanto a relevância de estudos envolvendo o construto da psicopatia em amostras brasileiras jovens.

O objetivo deste artigo é descrever resultados preliminares sobre a investigação de comportamentos interpessoais e aspectos não verbais da psicopatia mediante a utilização da IM-P, incluindo o processo de tradução/ adaptação do instrumento, o treinamento dos pesquisadores, a realização de um teste piloto e o estudo de confiabilidade interavaliadores. 


\section{Método}

O presente estudo integra um projeto de pesquisa mais amplo envolvendo a personalidade psicopática e populações jovens desenvolvido no Programa de Pós-Graduação em Psicologia da Pontifícia Universidade Católica do Rio Grande do Sul (PUCRS), Porto Alegre, RS. Foi realizado com adolescentes brasileiros em conflito com a lei que cumpriam medida socioeducativa de internação conforme preconiza o Estatuto da Criança e do Adolescente ${ }^{21}$.

Trata-se de estudo transversal, descritivo e correlacional. Os participantes estavam internados em uma unidade da Fundação de Assistência Socioeducativa do Rio Grande do Sul (FASE), localizada na Região Metropolitana de Porto Alegre (RS). A coleta de dados para este estudo ocorreu nos meses de outubro e novembro de 2008 .

\section{Apresentação do instrumento original}

No desenvolvimento da versão original da IM-P, os pesquisadores utilizaram, inicialmente, três fontes de informação: 1) revisão da literatura teórica e empírica relevante; 2 ) intuições clínicas baseadas nas impressões formadas pelos pesquisadores durante entrevistas com os detentos; e 3) levantamentos informais de investigadores da área de psicopatia em toda a América do Nor$t^{2}$. O objetivo era identificar as interações características das experiências clínicas com indivíduos psicopatas e transformá-las em conceitos operacionais, tentando criar itens com um nível intermediário de especificidade, a fim de possibilitar a avaliação dessas interações em um contexto de investigação.

A IM-P (versão original de Kosson et al., de 1993) ${ }^{2}$ consiste em uma escala de 21 itens projetada para avaliar o comportamento não verbal exibido pelo participante e os processos interpessoais evidentes que se estabelecem entre o participante e o entrevistador durante uma entrevista semiestruturada. Os itens são pontuados com base em aspectos específicos que servem de referência para sua observação, levando em conta a frequência e a intensidade com que se evidenciam durante a interação interpessoal.

A IM-P é uma medida coadjuvante que complementa instrumentos já existentes, como o PCL:YV, utilizando um método diferente das escalas Hare, priorizando as diferenças individuais e qualitativas ${ }^{9}$. É, portanto, baseada exclusivamente no funcionamento interpessoal observado na entrevista e nas impressões subjetivas do avaliador, ao passo que as escalas Hare se baseiam em respostas a perguntas específicas e informações colaterais. A IM-P não depende, portanto, da história prévia, refletindo a frequência com que determinados comportamentos e reações ocorrem no momento atual.

\section{Procedimentos preliminares para a gera- ção da versão brasileira da IM-P}

\section{Tradução}

A tradução da versão original da IM-P do inglês para o português, adaptada com base nas recomendações de Beaton et al. ${ }^{22}$, foi realizada por dois psicólogos e dois psiquiatras proficientes em língua inglesa. Cada um realizou uma tradução independente, resultando em quatro versões, as quais foram comparadas entre si e integradas, originando uma única versão consensual em português do Brasil. Dois juízes independentes retrotraduziram a versão em português, chegando por consenso a uma única versão em inglês. Essa nova versão em inglês foi apresentada a um dos autores da escala original para análise e ajustes de tradução. Posteriormente, o instrumento em português foi distribuído a quatro estudantes de doutorado em psicologia para verificar se eram capazes de compreender os itens. A versão final da IM-P, devidamente aprovada pelos autores, consiste na escala final em português do Brasil utilizada neste estudo (Vasconcelos SJC, Davoglio TR, Ronchetti R, Gauer GJC, Medida Interpessoal de Psicopatia, 2008, documento não publicado; ver lista de itens avaliados pela IM-P no Anexo 1).

\section{Treinamento da equipe de pesquisa}

A entrevista semiestruturada utilizada na aplicação do PCL:YV ${ }^{8}$ foi também norteadora para a aplicação da IM-P, a exemplo de outros estudos internacionais. Para a aplicação do PCL:YV, os pesquisadores já haviam sido treinados por um dos autores do instrumento original, professor convidado a vir ao Brasil pelo grupo de pesquisa no qual este projeto está inserido.

A observação de alguns pesquisadores ${ }^{18}$ de que seria conveniente a pontuação da IM-P sem que o avaliador tivesse conhecimento do desempenho do participante nos demais instrumentos, visando preservá-la de possíveis contaminações, apontaram a necessidade de a IM-P e instrumentos como o PCL:YV não serem pontuados pelo mesmo avaliador. No entanto, a legislação e as condições físicas de pesquisa no Brasil dificultam a utilização de recursos de filmagem e gravação, não sendo esta uma prática usual em situações de avaliação, especialmente com a população menor de idade institucionalizada cumprindo medida de segurança/proteção, diferentemente de outros países. Diante dessas questões, decidiu-se por realizar a coleta de dados ao vivo, ou seja, com a presença de dois pesquisadores em cada entrevista, ainda que isso pudesse implicar outros vieses. 
As instruções iniciais fizeram menção à presença do observador, estendendo a ele todas as responsabilidades éticas inerentes ao processo, porém com ênfase ao papel do entrevistador na condução da entrevista.

O treinamento ao qual os pesquisadores se submeteram considerou a dificuldade de identificação e a relevância da observação objetiva e minuciosa dos aspectos não verbais sutis que emergem durante a entrevista com indivíduos que apresentam traços de psicopatia, visando minimizar os efeitos da subjetividade do avaliador. Utilizando material audiovisual e entrevistas reais veiculadas na mídia, os pesquisadores foram instruídos a pontuar os itens da IM-P. Em seguida, as pontuações eram discutidas em relação ao que era proposto em cada item da escala.

\section{Teste piloto}

Foram realizadas cinco entrevistas piloto com a amostra para testar os procedimentos e ajustar as pontuações entre os juízes antes do início do estudo formal (tais pontuações não foram computadas na amostra final do estudo). Além de calibrar a utilização do instrumento, o teste piloto permitiu a escolha do local mais adequado para a realização das entrevistas nas dependências da instituição, bem como o posicionamento físico mais favorável de cada pesquisador em relação ao participante. O posicionamento escolhido foi aquele que deixou o entrevistador frente a frente com o participante, e o(s) observador(es) em um plano de fundo, levemente mais afastado, com visão privilegiada de ambos, mas sem enfatizar sua presença ou obstruir a visão lateral do participante.

Optou-se também por não realizar as entrevistas nos consultórios de atendimento médico/psicológico ou na sala da assistência social da instituição socioeducativa, por duas razões: primeiro, porque ficavam muito próximas de áreas de circulação de técnicos, podendo gerar desconforto ou suspeita no participante quanto à confidencialidade das entrevistas; segundo, porque os adolescentes demonstraram uma atitude mais descontraída e receptiva fora desses espaços onde habitualmente comparecem para atividades de rotina da instituição.

\section{Amostra}

O estudo de confiabilidade interavaliadores para a IM-P foi realizado com 20 adolescentes masculinos em conflito com a lei, internados em uma unidade da FASE, localizada na Região Metropolitana de Porto Alegre. Os participantes foram recrutados pelos monitores institucionais a partir da lista de internos da instituição e aceitaram participar voluntariamente, sem nenhum ganho direto, concordando em assinar o termo de consentimento livre e esclarecido após receberem todas as informações acerca da sua inclusão na pesquisa.

Foram considerados critérios de inclusão: estar em medida socioeducativa em privação de liberdade; ter disponibilidade para realizar a entrevista sem comprometer as rotinas institucionais; e aceitar voluntariamente o convite para participar do estudo. Os critérios de exclusão consistiram na presença de sinais e sintomas psicóticos, retardo mental ou deficiência auditiva, clinicamente reconhecidos, ou na recusa em participar de qualquer estágio da coleta de dados.

\section{Instrumentos}

Para esta etapa da pesquisa, foram utilizados, além da IM-P, o PCL:YV e um questionário sociodemográfico, descritos a seguir.

a) Medida Interpessoal de Psicopatia (IM-P). A escala foi pontuada durante a realização da entrevista semiestruturada recomendada para a aplicação do PCL:YV8, com duração aproximada de 90 minutos. Um entrevistador conduziu a entrevista, enquanto os avaliadores observavam. Por meio da entrevista, foram investigados aspectos educacionais, laborais, hábitos e rotinas, história criminal, relacionamento familiar e relações íntimas do entrevistado. Para cada um dos 21 itens da IM-P, o avaliador estimou o número de vezes que a interação ou o comportamento de interesse ocorreu durante a entrevista, considerando a consistência e a intensidade com que se apresentavam. Depois, decidia a pontuação, marcando a que melhor descrevia a atitude ou interação interpessoal entre o indivíduo e o entrevistador: 1) não se aplica, quando o sentimento ou reação nunca ocorreu, ou de maneira alguma era típica durante a interação; 2) aplica-se em parte, quando o sentimento ou reação era evidente até certo ponto, ou raramente era apresentado; 3) aplica-se bem, quando o sentimento ou reação estava presente de modo significativo ou era frequentemente apresentado durante sua interação; 4) aplica-se completamente, quando o sentimento ou reação era claramente evidente, apresentado de forma consistente ou frequentemente. O escore total foi obtido pela soma simples da pontuação de cada item.

b) Inventário de Psicopatia de Hare (PCL:YV) ${ }^{\mathbf{8}}$. Adaptado do $P C L-R^{5}$, o $P C L: Y V$ é um instrumento composto por 20 itens, desenvolvido especificamente para avaliar a psicopatia em adolescentes, preenchido por um perito-avaliador. A pontuação se baseia em uma entrevista semiestruturada e em informações colaterais provindas de diversas 
fontes (prontuários, equipe institucional, familiares), que fornecem dados acerca dos diversos domínios da escala. O PCL:YV já foi traduzido para o português brasileiro e vem sendo utilizado em contextos de pesquisa, com resultados psicométricos preliminares e de confiabilidade bastante satisfatórios $(W=0,93 ; p<0,001)^{23}$.

c) Questionário sociodemográfico. Este instrumento foi utilizado para coletar informações dos participantes acerca de idade, escolaridade, situação familiar, renda, histórico clínico e jurídico.

\section{Procedimentos éticos e de coleta de dados}

Os autores que detêm os direitos autorais da IM-P original autorizaram sua utilização para fins de pesquisa e a posterior validação do instrumento para o português do Brasil. Em seguida, o projeto de pesquisa foi submetido e aprovado pelo Comitê de Ética em Pesquisa e Comissão Científica da PUCRS. Finalmente, obteve-se autorização legal para a realização da coleta de dados junto à FASE, bem como para a utilização de suas instalações, sempre em horários pré-agendados e de forma a inteferir o mínimo possível nas rotinas institucionais e atividades dos adolescentes. Todos os protocolos de segurança para ingresso na unidade foram rigorosamente cumpridos.

As entrevistas individuais foram realizadas sem nenhum ganho direto e imediato para os participantes e somente com os adolescentes que aceitaram participar. Os pesquisadores tomaram rigorosos cuidados a fim de preservar o sigilo das informações e a confidencialidade dos dados, especialmente aqueles relacionados à identificação dos adolescentes, sendo essas informações codificadas para o manuseio estatístico e os dados originais deletados.

$O$ estudo de confiabilidade interavaliadores envolveu a presença de três avaliadores e um entrevistador em cada entrevista, todos graduados em Psicologia ou Psiquiatria. Cada avaliador pontuou a IM-P independentemente, e o entrevistador pontuou o PCL:YV. Todas as análises estatísticas foram realizadas utilizando-se o Statistical Package for the Social Sciences (SPSS) for Windows, versão 16.0.

\section{Resultados}

\section{Características da amostra}

A idade média dos participantes foi de 16 anos e 7 meses (desvio padrão $=1,13$ ). A maioria $(60 \%$ ) era de cor branca e completou apenas a $6^{a}$ série do ensino fundamental $(80 \%)$, sendo que $90 \%$ foram reprovados na escola ao menos uma vez. Antes da internação, apenas $35 \%$ dos adolescentes entrevistados residiam junto com o pai e a mãe. A renda familiar média, para os participantes que a declararam (40\%), foi de 1,3 salário mínimo (desvio padrão $=1,65$ ). A medida socioeducativa com restrição de liberdade era decorrente da prática de infrações variadas; porém, para $50 \%$ dos participantes, a punição foi devida à prática de assalto com uso de armas, e para $25 \%$, por tentativa de homicídio ou latrocínio. A medida socioeducativa estava sendo cumprida pela primeira vez pela maioria dos participantes (70\%).

\section{Confiabilidade interavaliadores}

Os resultados estatísticos obtidos através do coeficiente de concordância de Kendall revelaram grau de concordância interavaliadores satisfatório para os escores totais da IM-P ( $W=0,84 ; p<0,001)$. Os 21 itens apresentaram coeficientes de concordância que variaram de 0,33 a 1, como se pode observar na Tabela 1 .

Tabela 1 - Concordância interavaliadores para a IM-P

\begin{tabular}{lcc}
\hline IM-P & $\mathbf{W}$ & $\mathbf{p}$ \\
\hline Item 1 & 0,68 & 0,004 \\
Item 2 & 0,51 & 0,063 \\
Item 3 & 0,79 & 0,001 \\
Item 4 & 0,65 & 0,007 \\
Item 5 & 0,81 & 0,001 \\
Item 6 & 0,78 & 0,001 \\
Item 7 & 0,33 & 0,457 \\
Item 8 & 0,45 & 0,126 \\
Item 9 & 0,71 & 0,003 \\
Item 10 & 0,65 & 0,007 \\
Item 11 & 0,001 \\
Item 12 & 1,00 & 0,092 \\
Item 13 & 0,48 & 0,017 \\
Item 14 & 0,60 & 0,001 \\
Item 15 & 0,75 & 0,004 \\
Item 16 & 0,69 & 0,015 \\
Item 17 & 0,61 & 0,001 \\
Item 18 & 0,75 & 0,004 \\
Item 19 & 0,68 & 0,059 \\
Item 20 & 0,51 & 0,001 \\
Item 21 & 0,76 & 0,041 \\
Total & 0,54 & 0,001 \\
\hline
\end{tabular}

É importante relatar que o resultado do item 7 foi gerado por um ajuste automático do SPSS, atribuindo-se o fato ao baixo valor de discordância do item. Tal resultado é compatível com a variabilidade muito pequena nas pontuações dos avaliadores neste item, além de uma baixa pontuação (somente um participante pontuou diferente e acima de 1 nesse item para todos os juízes). O resultado persistiu mesmo após a tentativa de corrigir o empate por meio de cálculo manual, utilizando fórmula matemática específica. Diante disso, foi realizado um novo cálculo para o coeficiente de concordância dos escores totais, com a remoção do item 7 . O cálculo para os escores totais também foi refeito após a remoção de outros dois itens ( 8 
e 12), que apresentaram concordância menor que 0,50. O resultado das análises estatísticas considerando essas providências é demonstrado na Tabela 2 .

Tabela 2 - Concordância Concordância interavaliadores para os escores totais da IM-P após a remoção de alguns itens

\begin{tabular}{lccc}
\hline $\mathbf{W}$ & $\mathbf{p}$ & $\mathbf{N}^{0}$ de itens & Itens excluídos \\
\hline 0,84 & 0,001 & 21 & Nenhum \\
0,86 & 0,001 & 20 & 7 \\
0,87 & 0,001 & 19 & $7 \mathrm{e} 8$ \\
0,87 & 0,001 & 18 & $7,8 \mathrm{e} 12$ \\
\hline
\end{tabular}

\section{Discussão}

\section{Procedimentos preliminares: tradução, trei- namento e teste piloto}

De acordo com a legislação específica do Conselho Federal de Psicologia ${ }^{19}$ para instrumentos de avaliação psicológica, os testes estrangeiros devem ser traduzidos para o português e adequados à realidade nacional a partir de estudos realizados com amostras brasileiras. Com base nessas normatizações, foi adotada neste estudo a tradução por especialistas, seguida da tradução reversa (retrotradução), como procedimento inicial para a tradução/adaptação da IM-P. Essa abordagem teve por objetivo minimizar a possibilidade de ênfase excessiva no retorno exato à língua original em detrimento das diferenças idiomáticas e culturais entre o português do Brasil e o inglês americano ${ }^{24}$. A comparação entre três diferentes traduções por especialistas gerou maior número de alternativas para a resolução de dificuldades intrínsecas ao processo de tradução. A tradução reversa, por sua vez, realizada por dois tradutores bilíngues independentes, oportunizou que os autores tivessem acesso a possíveis distorções na compreensão de algum item, podendo, assim, discuti-las com os pesquisadores.

Por outro lado, o treinamento prévio disponibilizado aos pesquisadores contribuiu substancialmente para o entendimento dos aspectos a serem observados na interação interpessoal e para a compreensão dos itens da IM-P. Além disso, considerando que a psicopatia é um construto que não figura nas atuais classificações diagnósticas para transtornos mentais ${ }^{10,25}$ e considerando também a diversidade de significados atribuídos ao termo, o treinamento foi essencial à familiarização da equipe de pesquisa com a descrição e definição da psicopatia $^{3,5,8}$ norteadora deste estudo.

\section{Confiabilidade interavaliadores}

Entre os estudos internacionais que utilizaram a IM-P em associação com diferentes versões do Inventário de
Psicopatia de Hare em amostras adultas, quatro ${ }^{2,18,19}$ avaliaram a confiabilidade interavaliadores por meio do coeficiente de correlação intraclasse, chegando a resultados que variaram de 0,60 a 0,83 para amostras de diferentes tamanhos. Em geral, tais estudos envolviam a gravação em vídeo da entrevista realizada com o participante, sendo os itens pontuados por um avaliador que assistia o material posteriormente. Porém, o meIhor resultado de confiabilidade nesses estudos foi obtido com a comparação da pontuação realizada pelo próprio entrevistador e por um avaliador que observava a entrevista ao vivo (como no estudo atual), com 0,83 para 27 entrevistas (estudo 1 descrito por Kosson et al. ${ }^{2}$ ) em uma amostra adulta. Observa-se, desse modo, que o coeficiente encontrado em nossa amostra de adolescentes brasileiros ( $\mathrm{W}=0,84$ ) pode estar relacionado também ao acompanhamento direto e ao vivo da entrevista, possibilitando uma maior visibilidade das interações interpessoais.

Neste estudo, a confiabilidade interavaliadores avaliada por três juízes apresentou um coeficiente de concordância considerado elevado ( $W=0,84 ; p<0,001$ ), mesmo sem a exclusão de nenhum item. Esse resultado não é apenas estatisticamente significativo, como também o é clinicamente, considerando que fatores interpessoais e não verbais são muito difíceis de serem mensurados objetivamente e sofrem grande impacto dos aspectos intrínsecos da própria interação que se estabelece durante a avaliação, assim como da personalidade e da subjetividade dos envolvidos no processo.

A maioria dos itens no estudo de confiabilidade da IM-P não apresentou problemas, porém alguns merecem considerações. O item 7 foi considerado o mais crítico, pois não foi possível obter uma variância nas respostas dos avaliadores para os 20 participantes compatível com o necessário para que fosse estatisticamente válido. Diante disso, foi realizado o cálculo da confiabilidade para os escores totais, considerando a exclusão do item 7 , elevando desse modo o resultado final para 0,86 ( $p$ $<0,001$ ).

O item 7 diz respeito ao participante "fazer solicitações ao entrevistador" por algo tangível, apontando para a possibilidade de que questões legais referentes à medida socioeducativa tenham contribuído para a pouca variabilidade observada em sua pontuação. É parte do regulamento institucional de adolescentes brasileiros em medida socioeducativa de internação não poderem receber nenhum objeto ou material de qualquer espécie de visitantes externos, sob pena de serem submetidos a sanções, ou mesmo as visitas entrarem na instituição carregando-os sem a devida autorização, ainda que sejam objetos simples, como canetas ou lápis. Desse modo, é intrínseco à condição desses adolescentes não 
solicitarem nada extraoficialmente, especialmente àqueles que não oferecem a segurança de acobertá-los caso venham a transgredir as normas. Assim, por ser um item que avalia uma questão importante referente ao funcionamento psicopático, antes da decisão de exclusão do item 7 da versão brasileira da IM-P, torna-se relevante averiguar como ele se comporta em amostras diferentes, que não estejam submetidas a normas institucionais ou regimes totalmente controlados ou fechados.

Os outros itens que apresentaram resultados mais baixos de concordância interavaliadores foram 0 item 8 ("tende a ser tangencial") e o item 12 ("perseveração"). Contudo, os resultados desses itens não podem ser explicados pelas mesmas razões do item 7. Assim, há a necessidade de maior observação desses itens em outras amostras ou em amostras maiores antes de se pensar em generalizações ou exclusões. De qualquer forma, considerando o resultado total da confiabilidade interavaliadores, o impacto da retirada isolada do item 7 $(\mathrm{W}=86)$ neste estudo mostrou-se mais significativo do que sua retirada associada aos outros dois itens citados $(\mathrm{W}=87)$.

\section{Considerações finais}

A psicopatia pode ser evidenciada por um estilo interpessoal disfuncional e típico que acompanha o comportamento antissocial, criminoso ou não, podendo ser identificada nas interações que a pessoa mantém com o entrevistador. A IM-P é um instrumento desenvolvido para avaliar diretamente essas interações, de modo sistemático e replicável.

Do ponto de vista das propriedades psicométricas da versão em português da IM-P, os resultados obtidos neste estudo indicam que a confiabilidade interavaliadores foi consistente e confiável. Embora as análises estatísticas apresentadas tenham apontado inconsistências isoladas para alguns itens da IM-P, de modo geral o grau de concordância encontrado foi elevado. Como já referido por Kosson et al. ${ }^{2}$, esses dados sugerem que a pontuação da IM-P não sofre influências significativas do viés do avaliador.

Considera-se, no entanto, que há diversas limitações neste estudo, sendo oportuna a utilização da IM-P em amostras mais robustas e diversificadas, inclusive quanto ao gênero e idade, podendo gerar análises estatísticas mais apuradas das propriedades psicométricas da escala. Isso permitiria, se necessário, a exclusão de itens com maior segurança e, possivelmente, a redução da escala, facilitando sua aplicação, porém sem prejuízos para a qualidade da medida. Além disso, os estudos internacionais publicados oferecem apenas dados de confiabilidade para a medida em amostras adultas, o que limitou a comparação dos atuais resultados.

Por outro lado, acredita-se que neste estudo o treinamento prévio oferecido aos pesquisadores sobre a utilização da escala mostrou-se válido e essencial para a homogeneidade da interpretação de cada item. Nesse sentido, a ausência de um manual de recomendações detalhadas sobre a descrição e pontuação dos itens também reforçou a utilidade do treinamento. Considera-se, ainda, a possibilidade de, em estudo futuros, utilizar-se a pontuação dos itens de 0 a 3 em vez de 1 a 4, como na versão original, o que já vem sendo praticado por alguns pesquisadores ${ }^{9,18}$.

É importante enfatizar que os resultados aqui descritos, embora relevantes, demandam cautela e continuidade da pesquisa no contexto brasileiro antes de serem considerados generalizáveis. Nesse sentido, alguns questionamentos foram suscitados a partir da pesquisa empírica em relação ao uso e à aplicabilidade da IM-P, demandando maior reflexão. A partir deles, sugere-se a continuidade da pesquisa no sentido de ajudar a determinar, por exemplo:

1. Se um único avaliador poderia pontuar tanto a IM-P quanto uma das versões do PCL sem gerar contaminações ou sobreposição de resultados, o que, no entanto, demandaria a gravação da entrevista em vídeo, o que ainda é uma prática a ser introduzida em países como o Brasil. Por questões práticas, considera-se que a utilização da coleta de dados ao vivo por avaliadores distintos para cada um dos instrumentos torna-se onerosa e nem sempre viável, em contextos tanto forenses quanto clínicos.

2. Se a aplicação da IM-P fora de uma situação tão formal quanto a proposta pela entrevista semiestruturada do PCL:YV (ou outras versões do PCL) poderia oferecer maiores informações sobre 0 comportamento interpessoal dos participantes. Sabe-se que as características de personalidade tendem a se apresentar de modo mais efetivo e intenso em situações cotidianas, nas quais o sujeito pode demonstrar mais abertamente seu estilo interpessoal típico ${ }^{17}$.

3. Se em amostras não carcerárias esses comportamentos interpessoais investigados pela IM-P poderiam emergir em maior intensidade e quantidade, tendo em vista os regulamentos disciplinares das instituições. Estudos com populações adolescentes que apresentam comportamentos antissociais e dificuldades interpessoais mas que não cumprem medida socioeducativa com privação de liberdade ou simplesmente não cumprem medida socioeducativa poderiam ser bastante úteis nesse sentido. 
4. Se a pouca habilidade das personalidades psicopáticas para empatizar e decodificar aspectos não verbais e afetivos em outras pessoas poderia se refletir nos resultados da IM-P. Sabe-se que os déficits receptivos para detectar reações emocionais de baixa intensidade, como medo, raiva, tristeza e ansiedade, típicos da psicopatia, têm sido alvo de diversas pesquisas ${ }^{1,26}$. Uma possibilidade, então, seria associar a IM-P a alguma medida desses déficits receptivos, a fim de correlacionar os resultados.

\section{Agradecimentos}

À Fundação de Assistência Socioeducativa do Rio Grande do Sul pela disponibilidade em apoiar a coleta de dados. Ao Conselho Nacional de Desenvolvimento Científico e Tecnológico (CNPq) e à Coordenação de Aperfeiçoamento de Pessoal de Nível Superior (CAPES) pelo apoio financeiro à pesquisa. À Profa. Dra. Jandyra Fachel, da Universidade Federal do Rio Grande do Sul (UFRGS), pelo suporte estatístico. Ao Prof. Dr. David S. Kosson, da Rosalind Franklin University of Medicine and Science, pela inestimável parceria.

\section{Referências}

1. Steuerwald B, Kosson DS. Emotional experiences of the psychopath. In: Gacono CB, ed. The clinical and forensic assessment of psychopathy: a practitioner's guide. Mahwah: Lawrence Erlbaum Associates; 2000. p. 111-35.

2. Kosson DS, Forth E, Steuerwald BL, Kirkhart KJ. A new method for assessing the interpersonal behavior of psychopathic individuals: preliminary validation studies. Psychol Assess. 1997;9(2):89-101.

3. Cleckley H. The mask of sanity. St. Louis: Mosby; 1941-1988.

4. Hare RD. The Hare Psychopathy Checklist-Revised. Toronto: Multi-Health Systems; 1991.

5. Hare RD. Manual for the Psychopathy Checklist-Revised. Toronto: Multi-Health Systems; 2003.

6. Farrington DP. The importance of child and adolescent psychopathy. J Abnorm Child Psychol. 2005;33(4):489-97.

7. Walsh T, Walsh Z. The evidentiary introduction of Psychopathy Checklist-Revised assessed psychopathy in U.S. courts: extent and appropriateness. Law Hum Behav. 2006;30:493-507.

8. Forth $A E$, Kosson DS, Hare RD. Manual for the Hare Psychopathy Checklist: Youth Version. Toronto: Multi-Health Systems; 2003.

9. Vitacco MJ, Kosson DS. Understanding psychopathy through an evaluation of interpersonal behavior: testing the factor structure of the Interpersonal Measure of Psychopathy in a large sample of jail detainees. Psychol Assess. 2010;3:638-49.

10. Associação Psiquiátrica Americana. Manual Diagnóstico e Estatístico de Transtornos Mentais - 4a edição - Revista (DSM-IV-TR). Porto Alegre: Artmed; 2002.
11. Bornstein RF. Behaviorally referenced experimentation and symptom validation: a paradigm for 21 st-century personality disorder research. J Pers Disord. 2003;17:1-18.

12. Klonsky ED, Oltmanns TF, Turkheimer NE. Informant-reports of personality disorder: relation to self-reports and future research directions. Clin Psychol (New York). 2002; 9:300-11.

13. Oltmanns T, Turkheimer E, Strauss M. Peer assessment of personality traits and pathology in female college students. Assessment. 1998; 5:53-65.

14. Leising $D$, Sporberg $D$, Rehbein D. Characteristic interpersonal behavior in dependent and avoidant personality disorder can be observed within very short interaction sequences. J Pers Disord. 2006;20(4):319-30.

15. Forth AE. Psychopathy and young offenders: prevalence, family background and violence. Program Branch Users Report. Ottawa: Ministry of the Solicitor General of Canada; 1995.

16. Bauer D. Psychopathy in incarcerated adolescent females: prevalence rates and individual differences in cognition, personality and behavior [doctoral dissertation]. North Chicago: Finch University of Health Sciences; 1999.

17. Kosson DS, Cyterski TD, Steuerwald BI, Newmann CS, Walker-Matthews S. Reliability and validity of the Psychopathy Checklist: Youth Version (PCL:YV) in nonincarcerated adolescent males. Psychol Assess. 2002;14:97-109.

18. Vassileva J, Kosson DS, Abramowitz C, Conrod P. Psychopathy versus psychopathies in classifying criminal offenders. Legal Criminol Psychol. 2005;10(1):27-43.

19. ZolondekS, Lilienfeld SO, PatrickCJ, Fowler KA. The Interpersonal Measure of Psychopathy: construct and incremental validity in male prisoners. Assessment. 2006;13(4):470-82.

20. Conselho Federal de Psicologia. Resolução 002/2003. http:// www.pol.org.br/pol/export/sites/default/pol/legislacao/ legislacaoDocumentos/resolucao2003_2.pdf. Acessado jun 2009.

21. Brasil. Estatuto da Criança e do Adolescente. http://www. planalto.gov.br/ccivil_03/leis/L8069.htm. Acessado jan 2009.

22. Beaton DE, Bombardier C, Guillemin F, Ferraz MB. Guidelines for the process of cross-cultural adaptation of self-report measures. Spine (Phila Pa 1976). 2000;25(24):3186-91.

23. Ronchetti R, Davoglio TR, Silva RS, Vasconcellos SJL, Gauer GJC. Inventário de Psicopatia de Hare: Versão Jovens (PCL:YV): estudo preliminar em amostra adolescente brasileira. Interam J Psychol. 2010;44(3):536-42.

24. Sandoval J, Durán RP. Language. In: Sandoval J, Frisby CL, Geisinger KF, Scheuneman JD, Grenier JR, eds. Test interpretation and diversity: achieving equity in assessment. Washington: American Psychological Association; 1998. p. 181-211.

25. Organização Mundial da Saúde. Classificação Estatística Internacional de Doenças e Problemas Relacionados à Saúde (CID-10). 10a ed. Porto Alegre: Artes Médicas; 2007.

26. Kosson DS, Suchy Y, Mayer A. Facial affect recognition in criminal psychopaths. Emotion. 2002;2(4):398-441.

\section{Correspondência}

Tárcia Rita Davoglio

Departamento de Pós-Graduação em Psicologia

Faculdade de Psicologia, PUCRS

Av. Ipiranga, 6681, prédio $11,9^{\circ}$ andar, sala 93

90619-900 - Porto Alegre, RS - Brasil

Tel.: (51) 3320.3550, ramal 7735

E-mail: tarciad@gmail.com 


\section{Anexo 1}

\section{Itens avaliados na Medida Interpessoal de Psicopatia (IM-P), versão em português brasileiro}

1. Interrupções

2. Recusa em tolerar interrupções

3. Desrespeita limites profissionais

4. Desrespeita limites pessoais

5. Testa o entrevistador

6. Faz comentários pessoais

7. Faz solicitações ao entrevistador

8. Tende a ser tangencial

9. Evita lacunas

10. Tranquilidade ou descontração atípica

11. Frustração diante do não confrontamento

12. Perseveração

13. Superioridade ética

14. Narcisismo explícito

15. Alusão ao entrevistador em histórias pessoais

16. Busca por aliança

17. Comportamento dramático

18. Irritação

19. Respostas impulsivas

20. Valentia expressa

21. Contato intenso do olhar 